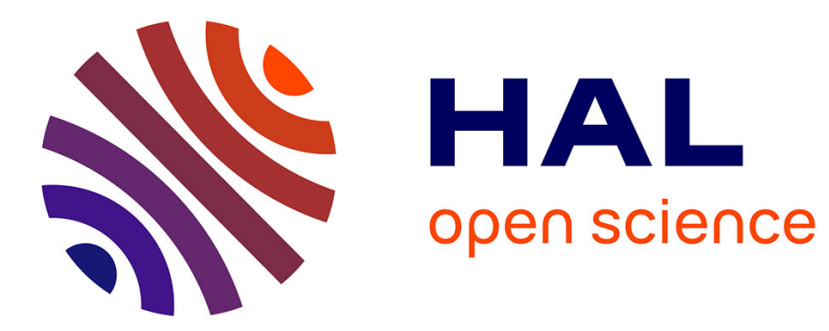

\title{
Climatisation en pays chauds et secs par toiture diode
} C.N. Awanou, P.F. Kieno, X. Berger

\section{To cite this version:}

C.N. Awanou, P.F. Kieno, X. Berger. Climatisation en pays chauds et secs par toiture diode. Revue de Physique Appliquée, 1987, 22 (6), pp.413-423. 10.1051/rphysap:01987002206041300 . jpa-00245556

\section{HAL Id: jpa-00245556 https://hal.science/jpa-00245556}

Submitted on 1 Jan 1987

HAL is a multi-disciplinary open access archive for the deposit and dissemination of scientific research documents, whether they are published or not. The documents may come from teaching and research institutions in France or abroad, or from public or private research centers.
L'archive ouverte pluridisciplinaire HAL, est destinée au dépôt et à la diffusion de documents scientifiques de niveau recherche, publiés ou non, émanant des établissements d'enseignement et de recherche français ou étrangers, des laboratoires publics ou privés. 
Classification

Physics Abstracts

$44.10-44.25-44.90$

\title{
Climatisation en pays chauds et secs par toiture diode
}

\author{
C. N. Awanou $(*)$, P. F. Kieno $(* *)$ et X. Berger $(* * *)$ \\ (*) Laboratoire de physique du rayonnement F.A.S.T.-U.N.B., B.P. 526 Cotonou, Bénin \\ (**) Institut de Mathématiques et de Physique, Université de Ouagadougou, B.P. 7021 Ouagadougou, \\ Burkina-Faso \\ (***) C.N.R.S., Laboratoire d'Ecothermique, B.P. 06561, Valbonne Cedex, France
}

(Reçu le 29 septembre 1986, accepté le 9 février 1987)

Article de mise au point.

\begin{abstract}
Résumé. - Les climats chauds et secs sont caractérisés par des écarts de températures très importants entre le jour et la nuit, et des températures de rayonnement du ciel très basses. L'article présente un dispositif de climatisation passive permettant d'assurer le confort thermique dans l'habitat de ces régions: la toiture « diode ». L'étude des modes de transferts thermiques au travers de cette toiture a été faite. Les résultats de l'expérimentation sur une cellule-test sont aussi présentés.
\end{abstract}

\begin{abstract}
The hot and arid countries are characterized by a very important difference between daytime and nighttime temperatures. The equivalent sky radiation temperature is very low. The present text describes a passive cooling system for enjoying a good thermal comfort in building in those countries : the « diode " roof. The results concerning the study about heat transfer and about the experimentation on a cell-test are presented.
\end{abstract}

\section{Introduction.}

En pays chauds, les modes d'échanges thermiques les plus usités pour les systèmes de climatisation passive sont: le rayonnement, la convection et l'évaporation. Les deux derniers sont relatifs à l'air ambiant, et donc dépendants des paramètres « agitation » et « débit ». Mais ils sont par contre inséparables des inconvénients «poussières », «bruits », « odeurs », ...

Le rayonnement solaire et le rayonnement de la voûte céleste sont les deux principales sources énergétiques dont les effets s'opposent dans ces pays chauds et secs, et desquelles dépendent les puissances des autres. Les dispositifs de climatisation rencontrés sont limités en efficacité d'une part par la faiblesse de la puissance radiative de la voûte céleste, d'autre part par la nécessité d'une protection contre les apports calorifiques d'origine solaire.

Les toitures constituées par de simples tôles, comme on en rencontre beaucoup en Afrique équatoriale, constituent des radiateurs chauds dans la journée, froids dans la nuit. Leur adoption ne résulte que de considérations de prix, et de solidité comparativement à ceux en chaume. L'amélioration apportée par certains est de disposer une plaque de contre-plaqué en dessous. Le volume perdu a une épaisseur voisine de celle des poutres, et est fermé pour diverses raisons (bruit provenant de la circulation naturelle de l'air, refuge d'animaux...). Le concept de toiture «diode » provient de la volonté de conserver une technique connue, et d'améliorer le comportement thermique du dispositif pour un prix modique :

* Renforcement de l'aspect isolant pour la journée en refusant le plus possible les apports solaires au moyen d'une peinture sélective, et en introduisant une masse dans la toiture faisant office de tampon thermique.

* Ajout d'un aspect conducteur pour la nuit par effet caloduc: les mouvements verticaux ascendant de l'eau évaporée contenue dans la toiture, et descendant gravifique du condensat engendrent un transfert de calories depuis l'intérieur de l'habitat vers l'extérieur (Fig. 1). Les frigories obtenues sont 


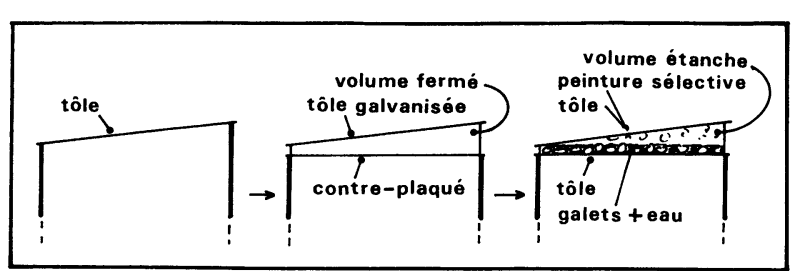

Fig. 1. - La toiture diode en tant qu'amélioration, à faible coût, d'un dispositif de toiture répandu en Afrique.

[The « diode» roof, as a cheap improvement of a wellknown african roof technique.]

stockées dans la masse inertielle qui les restituera avec un déphasage de plusieurs heures.

Le dispositif est très simple, et prend en compte les deux effets radiatifs opposés de l'échauffement solaire et du refroidissement par la voûte céleste. L'étude qui suit présente les recherches théoriques et expérimentales entreprises depuis plusieurs années pour la conception et la mise au point de ce système :

* La première partie présente une étude de la puissance radiative de la voûte céleste en fonction des conditions climatiques.

* Dans la seconde partie est décrit le dispositif de toiture diode, et sont présentées les équations exprimant les transferts thermiques.

* La troisième partie résume l'étude des transferts évapo-condensatifs pour la configuration adoptée du système.

* La quatrième partie est relative aux simulations théoriques sur ordinateur. L'influence d'un certain nombre de paramètres sur le comportement du dispositif pendant des cycles journaliers climatiques est analysée. Cette étude a conduit à une compréhension du fonctionnement et une optimisation du dispositif.

* La dernière partie présente les résultats expérimentaux obtenus avec une cellule-test.

\section{Caractéristiques climatiques des régions chaudes et sèches.}

Les régions chaudes et sèches sont éloignées de la mer, et, par suite, ne profitent pas des effets «tampon» résultant de l'évaporation et de la condensation de masses importantes d'eau, et des mouvements thermiques verticaux au sein de l'océan. La faible quantité de vapeur d'eau contenue dans l'atmosphère locale reste sensiblement constante au cours de la journée, ce qui se traduit dans le diagramme psychrométrique par un cycle hygro-thermique très allongé sur une ligne d'humidité absolue constante (Fig. 2) [1].

Par contre, en climat méditerranéen, le point de rosée n'est qu'à quelques degrés en dessous de la température de l'air, ce qui permet d'associer condensation et refroidissement radiatif : c'est le principe des revêtements sélectifs au blanc de chaux qui, par leur aspect micro-fissuré, permettent une imprégnation capillaire d'humidité dans les parois. L'évaporation diurne de cette humidité retarde l'échauffement. Les toitures à galets [2-3] ou à lit d'eau [4-5] procèdent de façon semblable.

En climat chaud et sec, le point de rosée ne peut être atteint aisément. Du fait de la rareté de l'eau
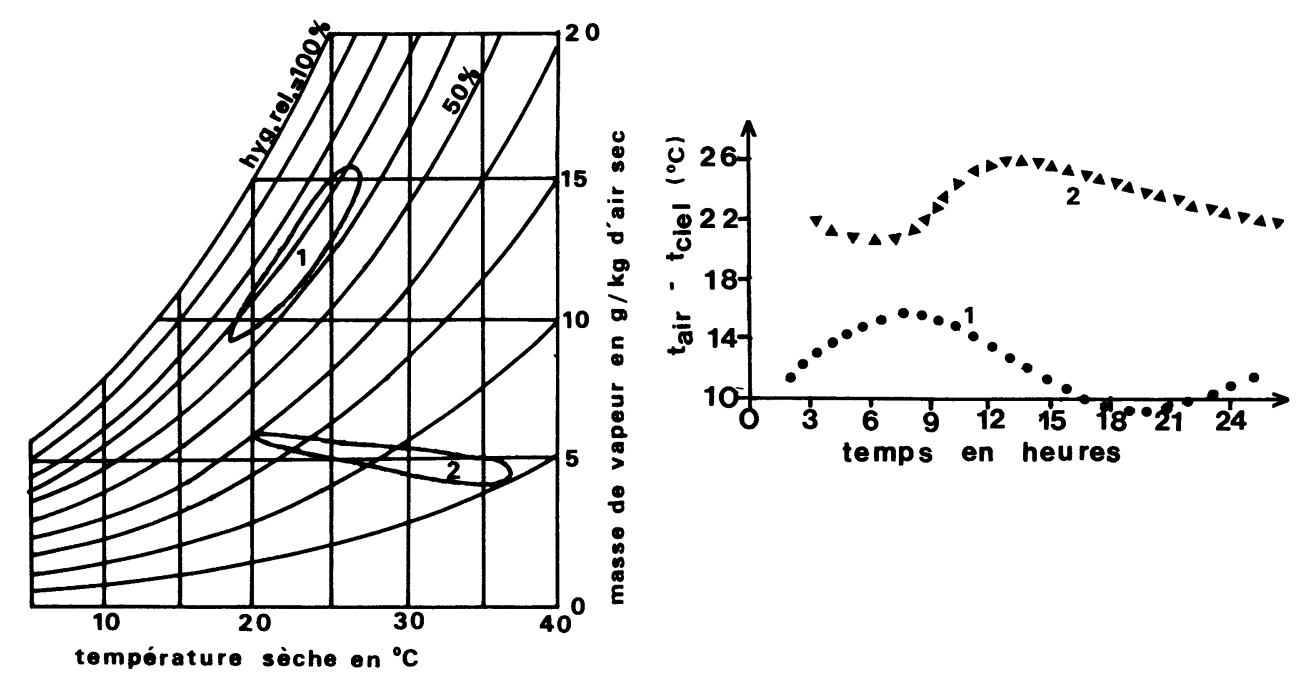

Fig. 2. - Cycle journalier climatique et écart $T_{\text {air }}-T_{\text {ciel }}$ en fonction du climat (1): $1:$ Nice (France) en juillet (climat méditerranéen) : humidité relative constante; $T_{\text {ciel }}$ minimale quand $T_{\text {air }}$ est minimale. $2:$ Kandi (Bénin) en février (climat continental) : poids d'eau par $\mathrm{m}^{3}$ d'air constant ; écart $T_{\text {air }}-T_{\text {ciel }}$ maximal quand $T_{\text {air }}$ est maximale.

[Climatic daily cycle and difference $T_{\text {air }}-T_{\text {sky }}$ as functions of the climate (1) : $1:$ Nice (France) in july (mediterranean climate): the relative hygrometry is almost constant ; $T_{\text {sky }}$ is minimum when $T_{\text {air }}$ is minimum. 2 : Kandi (Benin) in february (continental climate) : the water content is constant ; $T_{\text {air }}-T_{\text {sky }}$ is maximum when $T_{\text {air }}$ is maximum.] 
dans ces régions, il est obligatoire de rechercher des dispositifs simples d'évaporation et de condensation fonctionnant en ambiance fermée. L'introduction d'un échangeur supplémentaire diminuerait le rendement du système si des particularités climatiques à ces régions n'existaient pour compenser cette baisse, et si un avantage d'isolation n'était en même temps ajouté.

Les températures minimales basses résultent du faible rayonnement de la voûte céleste pouvant être assimilée à un corps froid. L'ensemble des couches atmosphériques, chacune avec sa transparence et son émissivité spectrales, constitue un corps dont le rayonnement est celui d'un corps noir à la température ambiante $T_{\text {air }}$, excepté dans la bande $(8-14 \mu)$ appelée «fenêtre atmosphérique». Dans cette bande, l'émission, plus faible, est principalement fonction de la teneur en vapeur d'eau. La température $T_{\text {ciel }}$ du corps noir énergétiquement équivalent peut être exprimée par la relation suivante [6] :

$$
T_{\text {ciel }}=\varepsilon_{\mathrm{c}}^{0,25} T_{\text {air cor. }} \quad \mathrm{K}
$$

avec :

$$
T_{\text {air cor. }}=T_{\text {air }}+K(H)+L(H)\left(T_{\mathrm{d}}-T_{\text {air }}\right)
$$

$\varepsilon_{\mathrm{c}}$ : émissivité

$T_{\mathrm{d}}$ : température de rosée $\mathrm{K}$.

$K(H)$ et $L(H)$ sont des paramètres dépendants d'un indice tri-horaire $H$ (Tabl. I)

Tableau I. - Coefficients $K$ et $L$ en fonction de l'indice tri-horaire $H$ (4 pour le lever du soleil...).

\begin{tabular}{l|rrrrrrrr}
$H$ & \multicolumn{1}{|c}{1} & \multicolumn{1}{c}{2} & \multicolumn{1}{c}{3} & \multicolumn{1}{c}{4} & \multicolumn{1}{c}{5} & \multicolumn{1}{c}{6} & \multicolumn{1}{c}{7} & \multicolumn{1}{c}{8} \\
\hline$K$ & 6,45 & 7,18 & 7,65 & 3,86 & 0,18 & 2,90 & 4,85 & 5,65 \\
$L$ & 0,435 & 0,620 & 0,616 & 0,399 & 0,276 & 0,290 & 0,311 & 0,364
\end{tabular}

Les coefficients $K$ et $L$ traduisent les influences de la température du sol relativement à celle de l'air, et de la hauteur de la couche d'inversion (l'humidité étant retenue entre le sol et cette couche). L'effet journuit est exprimé par la relation :

$$
\begin{aligned}
& \varepsilon_{\mathrm{c} \mathrm{nuit}}=0,770+0,0038\left(T_{\mathrm{d}}-273,15\right) \\
& \varepsilon_{\mathrm{c} \mathrm{jour}}=0,752+0,0048\left(T_{\mathrm{d}}-273,15\right) .
\end{aligned}
$$

Le diagramme psychrométrique (Fig. 2) a été utilisé pour montrer les situations des lignes :

$$
\begin{aligned}
T_{\text {air }}-T_{\text {ciel }} & =\text { Cte } & & \mathbf{K} \\
\sigma\left(T_{\text {air }}^{4}-T_{\text {ciel }}^{4}\right) & =\text { Cte } & & \text { W. } \mathbf{m}^{-2} .
\end{aligned}
$$

On observe que la puissance frigorifique en climat chaud et sec est importante. Plus particulièrement, c'est en milieu de journée que l'écart $T_{\text {air }}-T_{\text {ciel }}$ est le plus grand (Figs. 2 et 4 ). L'intérêt des peintures sélectives se trouve mis en évidence puisqu'en n'absorbant que très peu de rayonnement solaire, il est possible avant la nuit de porter la température d'une surface en dessous de la température ambiante.

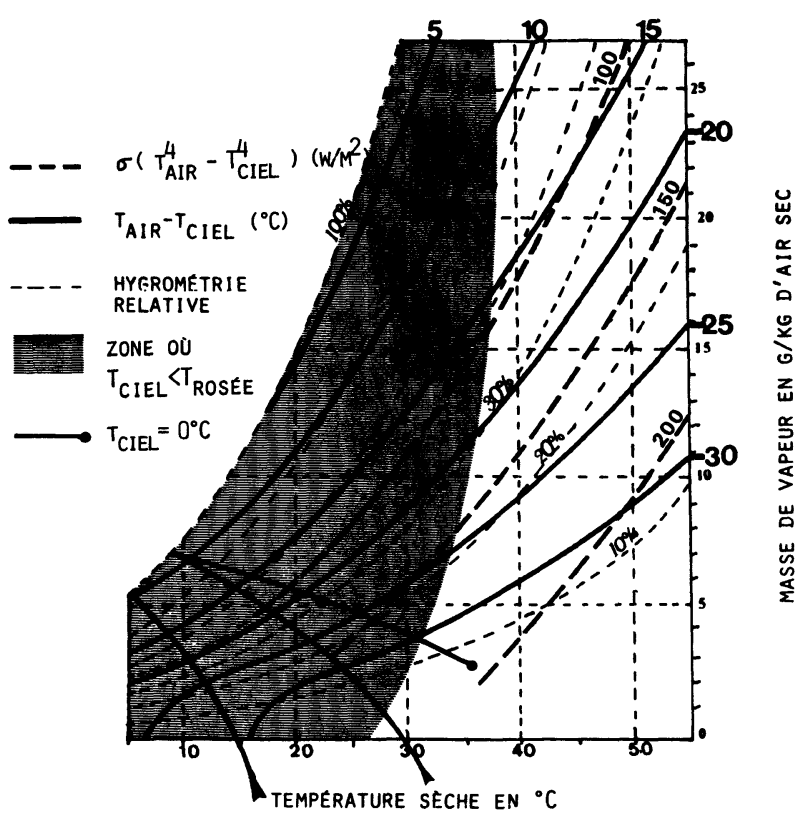

$\longrightarrow \sigma\left(T_{\text {AIR }}^{4}-T_{\text {CIEL }}^{4}\right)-2.8\left(T_{A I R}-273\right)^{1.25}=0:$ CONECTION NATURELE
$\sigma \sigma\left(T_{\text {AIR }}^{4}-T_{\text {CIEL }}^{4}\right)-10(1 \mathrm{M} / \mathrm{s})^{0.5}\left(T_{A I R}-273\right)=0:$ CONEECTION FORCEE

Fig. 3. - Propriétés radiatives du ciel clair exprimées dans le diagramme psychrométrique (1). En climat chaud et sec le point de rosée peut être difficilement atteint, mais la puissance frigorifique du ciel y est maximale.

[Radiative properties of clear skies drawn in the psychrometric diagram (1). In hot dry climates the dew point is hardly reached, but the cooling efficiency is maximum.]

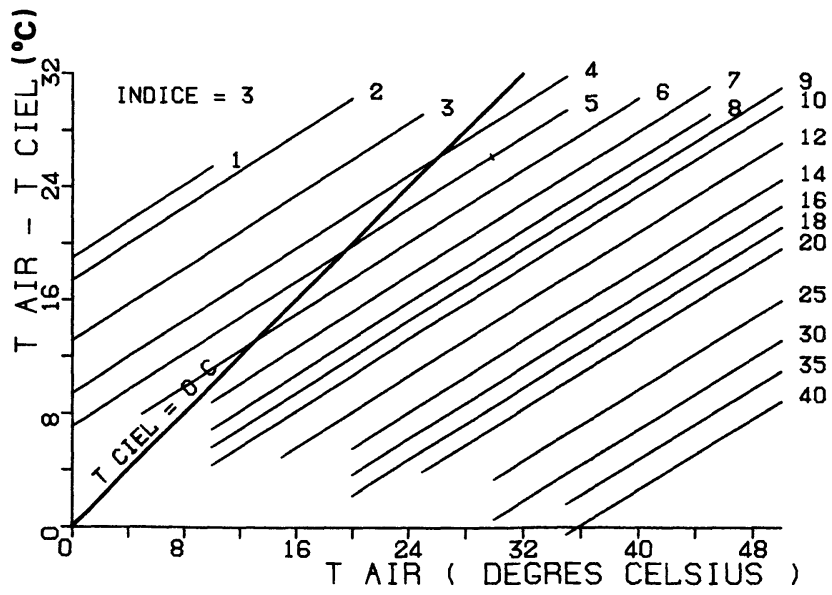

Fig. 4. - Ecart $T_{\text {air }}-T_{\text {ciel }}$ en fonction de la température d'air et de la quantité d'eau contenue dans l'air (en g/kg d'air sec) (1) : à teneur en eau constante, l'écart augmente avec $T_{\text {air }}$.

$\left[T_{\text {air }}-T_{\text {sky }}\right.$ as a function of air temperature and water content ( $\mathrm{g} / \mathrm{kg}$ of dry air) (1). For a constant water content, the difference increases with $T_{\text {air }}$ ] 
Les régions à climat chaud et sec sont aussi caractérisées par les deux traits importants suivants : présence possible dans l'atmosphère d'aérosols en quantité importante (poussières, brumes sèches, vents de sable comme l'harmattan ou le sirocco). La température équivalente de ciel s'en trouve élevée [7] et les possibilités de climatisation radiative restreintes, d'autant plus que l'état des surfaces recouvertes de peintures sélectives ne parvient pas à être maintenu toujours propre. Les toitures à revêtement sélectif ne conviennent pas à des habitats implantés dans ces régions à ciel pollué; même dans les régions à ciel clair, un entretien de l'état des surfaces assurant les échanges radiatifs est indispensable.

Une durée de nuit voisine de $12 \mathrm{~h}$ dans les régions tropicales est un paramètre favorable, absent en régions méditerranéennes.

\section{La toiture « diode ».}

2.1 CONCEPTION ET DESCRIPTION. - Le confort thermique en climat chaud incite l'homme à éviter les ambiances trop chaudes dans lesquelles son organisme a recours au processus fatigant de la sudation pour maintenir son équilibre thermique $[8$, 9]. Il est, de même, incité à éviter les ambiances qu'il juge trop froides ; l'adaptation de son organisme se fait sur des situations thermiques fonctions du climat moyen local [10]. Les écarts sont perçus avec plus de désagrément dans le froid que dans le chaud (Fig. 5).
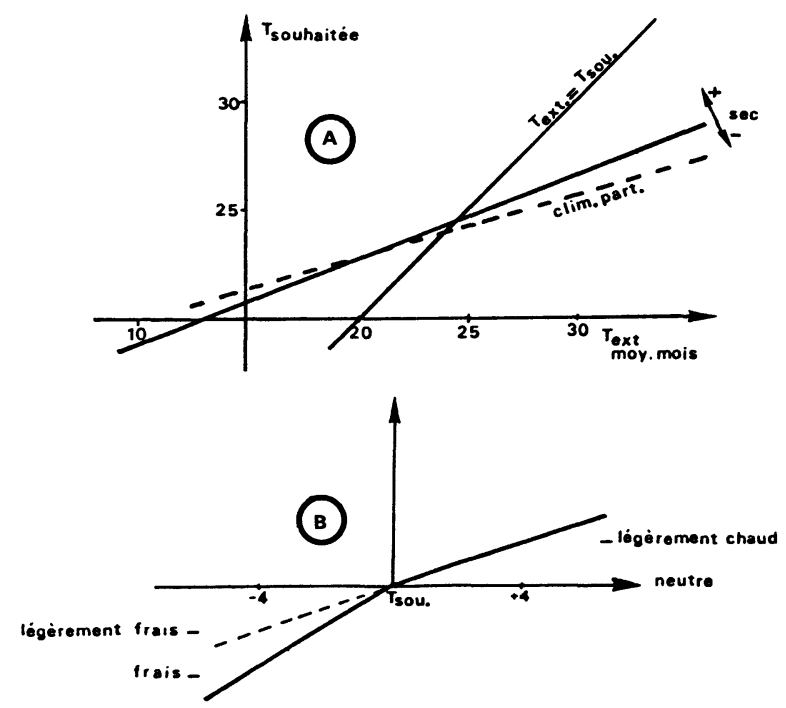

Fig. 5. - A : Température souhaitée par l'être humain en faible activité en fonction de la température extérieure moyenne mensuelle; influence de l'hygrométrie et de l'utilisation intermittente d'un climatiseur. B : sensibilité dans le froid plus grande que dans le chaud [8-9].

[A : wished temperature for a man in low activity, as a function of mean monthly outdoor temperature ; influences of hygrometry and of intermittent use of an air conditioning unit. B : sensitiveness in cold greater than in warm [8-9].]
Les possibilités de climatisation convective par ventilation nocturne d'air rafraîchi complètent celles de climatisation radiative obtenues en fin de journée - début de nuit dans les espaces semi-ouverts fort usités dans ces pays : cours, patios... Le but de la toiture diode est d'éviter tant les ambiances trop froides en fin de nuit rencontrées dans les volumes semi-ouverts, qu'en début de nuit des ambiances trop chaudes des volumes fermés. Les frigories récupérées par la toiture naturellement orientée vers le ciel doivent donc être stockées et restituées avec un déphasage temporel. L'objectif n'est pas d'obtenir des températures minimales très basses, mais plutôt de maintenir une ambiance de confort. Par ailleurs, la fraîcheur d'un plafond peut être grande sans qu'il ne soit ressenti de désagrément $[11,12]$.

Depuis l'intérieur de l'habitat jusque vers l'extérieur, les éléments constitutifs de la toiture diode sont (Fig. 6) :
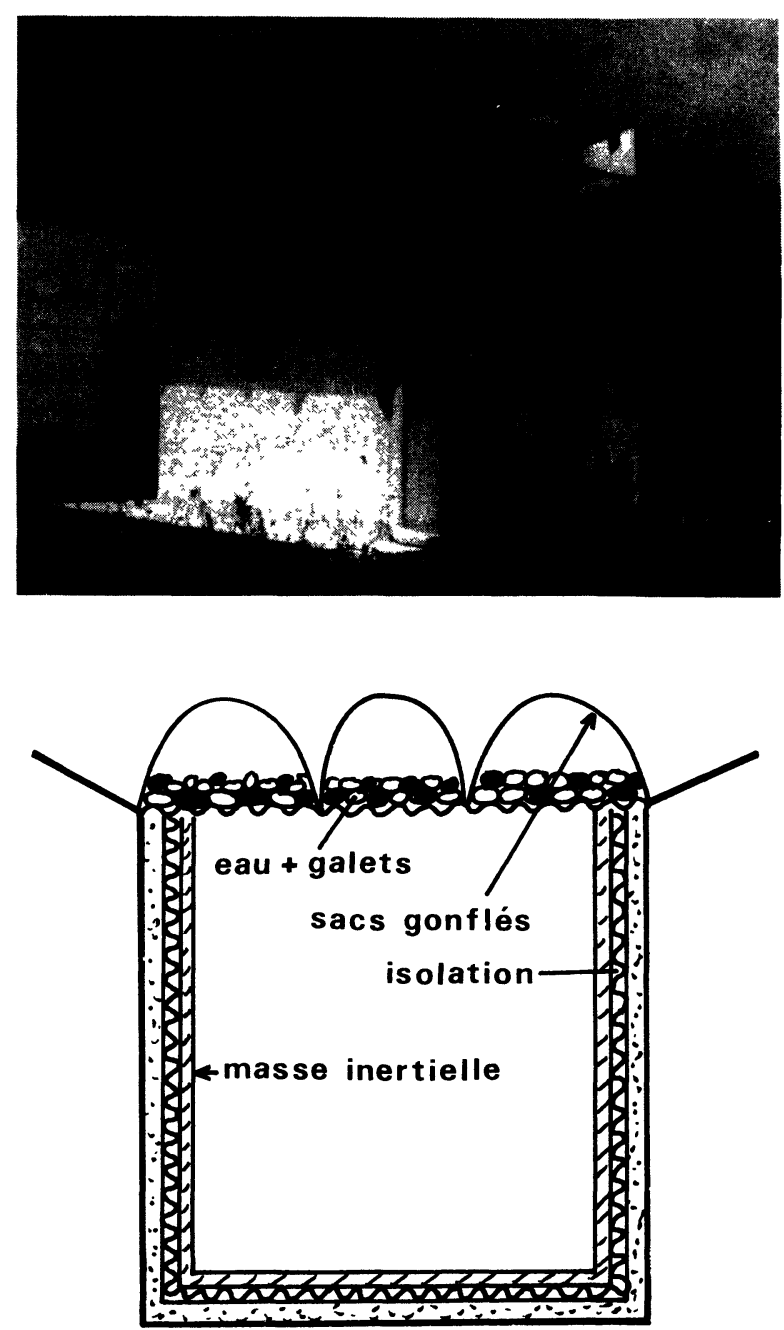

Fig. 6. - La cellule-test à toiture diode construite près de Nice : photo et schéma de principe.

[The « diode » roof cell test built near Nice : photographic picture and skeleton diagram.] 
* Le plafond de la pièce en tôle galvanisée, comme cela est répandu pour les constructions en pays chauds. La face, côté intérieur, est recouverte d'une peinture émissive pour favoriser les échanges radiatifs dans l'habitat.

* Un lit de galets d'environ $5 \mathrm{~cm}$ d'épaisseur avec un peu d'eau $\left(10 \mathrm{l} / \mathrm{m}^{2}\right)$ constitue la masse thermique pouvant stocker et véhiculer les frigories. Il est maintenu en un milieu fermé bien étanche. Une couche d'air de 8 à $10 \mathrm{~cm}$ d'épaisseur dans ce même milieu assure l'isolation diurne, mais devient conductrice lorsque le processus d'évapo-condensation fonctionne.

* Une tôle galvanisée fermant l'ensemble est maintenue tout autour par des tasseaux de bois. La peinture extérieure est d'un blanc sélectif froid pour limiter l'absorption du rayonnement solaire et favoriser les échanges radiatifs vers le ciel (par exemple, blanc de titane couramment répandu dans ces régions).

Pour l'expérience, des sacs en polyéthylène gonflés ont constitué une variante de réalisation. La forme non plane de l'échangeur supérieur est voulue pour faciliter l'écoulement interne du condensat.

La description ci-dessus constitue le principe de base du dispositif dont l'un des critères recherchés est un prix de revient faible (ressources économiques), et l'autre, une insertion facile dans le contexte d'habitat (matériaux de construction nécessaires disponibles presque partout ; maintien du style architectural). Des variantes peuvent être réalisées, comme par exemple :

- disposition de la masse thermique sous le plancher et liaison par une tuyauterie;

- réalisation de voûtes pleines appuyées sur des colonnes pour augmenter la surface d'échange vers l'habitat ;

- installation de protections solaires extérieures : stores à lames mobiles...;

- débordement de la toiture, à la fois pour protéger les murs verticaux et augmenter la surface radiative d'échange.

La nuit, l'échangeur supérieur est refroidi par rayonnement sur la voûte céleste, et par convection lorsqu'il est plus chaud que l'air ambiant. Le transfert de chaleur de l'intérieur de l'habitat vers l'extérieur est assuré par une partie de l'eau de la masse inertielle : l'évaporation entraîne une saturation en humidité de la couche d'air et une condensation sur la face intérieure de l'échangeur supérieur dont la température est plus basse. Le condensat redescend au plus bas de la masse thermique par gravité.

Dans la journée, l'échauffement solaire de l'absorbeur supérieur est limité par suite de faible valeur du coefficient $\alpha$ d'absorption de la peinture sélective. Il est principalement évacué vers l'extérieur par convection forcée (ventilation naturelle) sur l'air ambiant. Intérieurement aucune condensation ne peut se produire car l'absorbeur est plus chaud que l'air confiné. Il n'y a aucune évaporation possible puisqu'il ne peut y avoir de condensation. La couche d'air est immobile et se comporte comme un isolant.

2.2 EQUATIONS PRINCIPALES. - L'étude des échanges thermiques dans le système « diode » est faite en considérant le dispositif constitué de trois parties distinctes :

- le plafond assurant les échanges entre l'habitat et la masse thermique ;

- la masse thermique assurant un stockage et un transfert conductif d'une face à l'autre ;

- l'absorbeur supérieur, à inertie très faible, assurant les échanges entre la masse thermique et l'environnement extérieur.

La figure 7 représente sous forme schématique les différents transferts thermiques concernant le dispositif.

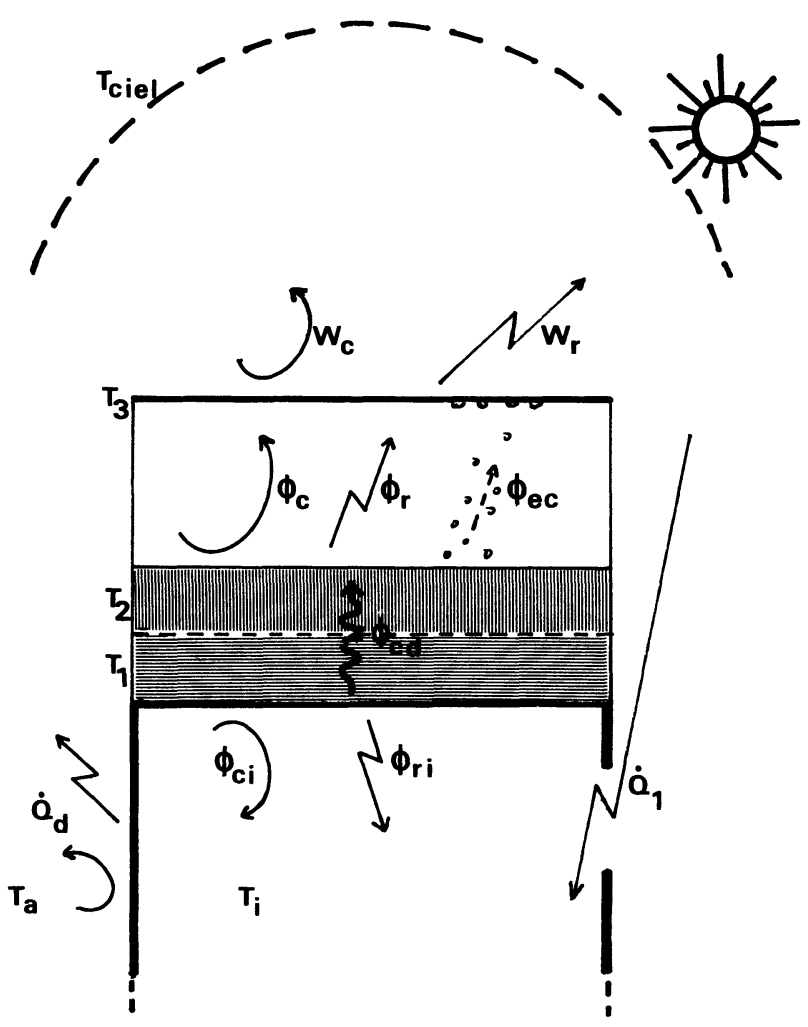

Fig. 7. - Les flux thermiques d'échange intervenant dans les différentes parties du dispositif de toiture diode.

[The thermal fluxes in the various parts of the diode roof system.]

2.2.1 Echanges thermiques au niveau du plafond. Une masse thermique $m_{\mathrm{i}}$ considérée sous forme de son équivalent eau $\left(C_{\mathrm{pe}}\right.$ : chaleur spécifique de l'eau), est introduite pour correspondre à la masse inertielle de l'intérieur de l'habitat. Elle rend compte de l'état thermique des parois. L'équation 
qui exprime l'échange thermique entre l'intérieur, à la température $T_{\mathrm{i}}$, et le plafond, à la température $T_{1}$ peut s'écrire :

$$
m_{\mathrm{i}} C_{\mathrm{pe}} \mathrm{d} T_{\mathrm{i}} / \mathrm{d} t=\dot{Q}_{\mathrm{d}}+\dot{Q}_{1}+S\left(\phi_{\mathrm{ri}}+\phi_{\mathrm{ci}}\right)
$$

avec :

$$
Q_{\mathrm{d}}=G^{*} V\left(T_{\mathrm{a}}-T_{\mathrm{i}}\right) \quad \mathrm{W} / \mathrm{m}^{2}
$$

$Q_{\mathrm{d}}$ : déperditions compte non tenu ^de la toiture, mais compte tenu du renouvellement d'air (d'où l'introduction du coefficient $G^{*}$ au lieu de $G$ [13]).

$V$ : volume de l'habitat $\mathrm{m}^{3}$

$T_{\mathrm{a}}$ : température de l'ambiance extérieure à l'habitat $\mathrm{K}$

$\dot{Q}_{1}$ : apports gratuits : éclairage, apports radiatifs solaires, présence éventuelle de personnes $\mathrm{W} / \mathrm{m}^{2}$

$$
\phi_{\mathrm{ri}}=\varepsilon_{1} \sigma\left(T_{1}^{4}-T_{\mathrm{i}}^{4}\right) \quad \mathrm{W} / \mathrm{m}^{2}
$$

$\phi_{\mathrm{ri}}$ : flux échangé radiativement avec une émissivité moyenne $\varepsilon_{1}$

$$
\phi_{\mathrm{ci}}=h_{\mathrm{ci}}\left(T_{1}-T_{\mathrm{i}}\right) \quad \mathrm{W} / \mathrm{m}^{2}
$$

$\phi_{\mathrm{ci}}$ : flux échangé convectivement avec le coefficient d'échange $h_{\mathrm{ci}}$

$$
\sigma=5,67 \times 10^{-8} \quad \text { W. m }{ }^{-2} \cdot \mathrm{K}^{-4}
$$

$\sigma$ : constante de Stefan-Boltzmann.

Les quantités sont comptées positivement dans le cas d'un apport à l'habitat, négativement dans le cas contraire.

\subsubsection{Echanges thermiques dans la masse thermique.}

- Le lit de galets-eau a été subdivisé en deux couches identiques, aux températures $T_{1}$ et $T_{2}$, entre lesquelles les échanges s'effectuent par conduction. L'équation (2) exprime que l'échauffement de la couche inférieure est fonction des apports calorifiques en provenance de l'habitat et de la couche supérieure :

$$
\begin{aligned}
1 / 2\left(m_{\mathrm{e}} C_{\mathrm{pe}}+m_{\mathrm{g}} C_{\mathrm{pg}}\right) \mathrm{d} & T_{1} / \mathrm{d} t= \\
& =-S\left(\phi_{\mathrm{ri}}+\phi_{\mathrm{ci}}+\phi_{\mathrm{cd}}\right)
\end{aligned}
$$

avec :

$m_{\mathrm{e}}:$ masse d'eau dans la toiture $\mathrm{kg}$

$m_{\mathrm{g}}$ : masse de galets dans la toiture $\mathrm{kg}$

$C_{\mathrm{pg}}$ : chaleur spécifique des galets $\mathrm{J} \mathrm{kg}^{-1} \mathrm{~K}^{-1}$

$S$ : surface du plafond $\mathrm{m}^{2}$

$$
\phi_{\mathrm{cd}}=\lambda\left(T_{1}-T_{2}\right) /(2 e) \quad \mathrm{W} / \mathrm{m}^{2}
$$

$\phi_{c d}:$ flux d'échange conductif entre les deux couches $\lambda \quad$ : conductivité thermique de l'ensemble galetseau W. $\mathrm{m}^{-1} \cdot \mathrm{K}^{-1}$

$e \quad$ : épaisseur de la couche galets-eau $\mathrm{m}$.

L'équation (3) exprime l'échauffement de la couche supérieure en fonction des apports conductif inférieur, convectif, radiatif et évaporatif supérieurs :

$$
\begin{aligned}
1 / 2\left(m_{\mathrm{e}} C_{\mathrm{pe}}+m_{\mathrm{g}} C_{\mathrm{pg}}\right) \mathrm{d} T_{2} / \mathrm{d} t= \\
=S\left(\phi_{\mathrm{cd}}-\phi_{\mathrm{c}}-\phi_{\mathrm{r}}-\phi_{\mathrm{ec}}\right)
\end{aligned}
$$

avec :

$$
\phi_{\mathrm{r}}=\varepsilon_{2} \sigma\left(T_{2}^{4}-T_{3}^{4}\right) \quad \mathrm{W} / \mathrm{m}^{2}
$$

$\phi_{\mathrm{r}}$ : flux d'échange radiatif $\left(\varepsilon_{2}:\right.$ émissivité moyenne des surfaces en regard)

$T_{3}$ : température de l'absorbeur supérieur $\mathrm{K}$

$\phi_{\mathrm{ec}}$ : flux d'échange par évapo-condensation $\mathrm{W} / \mathrm{m}^{2}$

$\phi_{\mathrm{c}}$ : flux d'échange convectif-conductif avec la lame d'air confiné $\mathrm{W} / \mathrm{m}^{2}$.

Ces trois flux sont fonctions de la valeur de $T_{3}$ relativement à $T_{2}$ :

$T_{3}<T_{2}$ : les surfaces en regard sont mouillées et $\varepsilon_{2}$ est voisin de 1 .

Le processus évapo-condensatif et la présence d'une paroi plus froide au-dessus du lit de galets-eau entraînent des mouvements convectifs de l'air confiné.

$T_{3}>T_{2}$ : les surfaces en regard sont partiellement humides et $\varepsilon_{2}$ peut être rendu nettement inférieur à 1 .

Le processus évapo-condensatif est inexistant et l'air confiné est en couches stratifiées. $\phi_{\mathrm{c}}$ devient un flux conductif faible.

2.2.3 Echanges thermiques au niveau de l'absorbeur supérieur. - L'équation (4) exprime le transfert d'énergie entre l'intérieur du dispositif et l'environnement extérieur, modulé par la présence de la masse thermique $m_{\mathrm{a}}$, bien que faible, de l'absorbeur :

$m_{\mathrm{a}} C_{\mathrm{pa}} \mathrm{d} T_{3} / \mathrm{d} t=S\left(\phi_{\mathrm{c}}+\phi_{\mathrm{r}}+\phi_{\mathrm{ec}}+W_{\mathrm{c}}+W_{\mathrm{s}}\right)$

avec :

$C_{\mathrm{pa}}$ : chaleur spécifique de l'absorbeur $\mathrm{J} \mathrm{kg}^{-1} \mathrm{~K}^{-1}$

$$
W_{\mathrm{c}}=h_{\mathrm{c}}\left(T_{\mathrm{a}}-T_{3}\right) \quad \mathrm{W} / \mathrm{m}^{2}
$$

$W_{\mathrm{c}}$ : flux échangé convectivement entre l'absorbeur et l'air ambiant (convection forcée) avec le coefficient d'échange $h_{\mathrm{c}}$

$$
W_{\mathrm{s}}=\alpha R+\varepsilon_{3} \sigma\left(T_{\text {ciel }}^{4}-T_{3}^{4}\right) \quad \mathrm{W} / \mathrm{m}^{2}
$$

$W_{\mathrm{s}}$ : flux d'échange radiatif avec l'extérieur 
$\varepsilon_{3} \quad$ : émissivité de la surface extérieure de l'absorbeur

$\alpha$ : coefficient d'absorption du rayonnement solaire

$R$ : flux incident du rayonnement solaire $\mathrm{W} / \mathrm{m}^{2}$.

2.2.4 Solution des équations. - Les quantités intervenant dans les équations ci-dessus sont des fonctions de paramètres dépendant de l'état du système ou des conditions climatiques instantanées. Leurs expressions détaillées [14] ne sont pas ici présentées pour ne pas alourdir le texte.

$\mathrm{Au}$ niveau des trois masses thermiques (les deux couches de galets-eau et l'absorbeur supérieur), chaque équation se décompose en deux de manière à considérer à chaque instant les flux entrants et ceux sortants. La détermination instantanée des quatre températures $T_{\mathrm{i}}, T_{1}, T_{2}$ et $T_{3}$ a été obtenue sur ordinateur Vax 750 en intégrant numériquement par pas d'une minute le système d'équations ci-dessus. Un jeu de clés binaires permet de modifier en cours d'intégration les expressions des diverses quantités ou paramètres. L'étude théorique et expérimentale est présentée dans les deux paragraphes ci-après. Elle a porté d'une part sur l'optimisation du processus caloduc (recherche de l'expression de la quantité d'eau évapo-condensée), d'autre part sur l'optimisation du dispositif placé dans un contexte climatique et en vue d'en obtenir un comportement donné.

\section{Le processus caloduc évapo-condensatif.}

Le bon fonctionnement de la toiture diode est basé sur une connaissance approfondie de ce mode d'échange.

3.1 ASPECTS THÉORIQUES. - L'évaporation d'une masse $m$ d'eau dans un gaz résulte de l'agitation moléculaire qui entraîne la diffusion de matière des zones à forte concentration vers celles à plus faibles concentrations. C'est la loi de Fick, analogue à la loi de Fourier pour les flux de chaleur :

$$
m=-D_{\mathrm{c}} \partial C / \partial z S
$$

avec :

$D_{c}$ : coefficient de diffusion de la vapeur du liquide dans le gaz $\mathrm{m}^{2} \mathrm{~s}^{-1}$

$\partial C / \partial z$ : gradient de concentration au voisinage de l'interface $D_{\mathrm{c}}$ est déterminé par la relation de Schirmer [15]. La méconnaissance de $\partial C / \partial z$ amène à transformer la relation (5) pour faire apparaître le gradient de pression. L'intégration de l'équation obtenue aboutit à l'équation de Stefan. En considérant linéaires les gradients de pression dans la couche limite (épaisseur $l_{0}$ ) et le fait que les pressions partielles de vapeur $P_{v}$, hors de la couche limite, et saturante $P_{2}$ (couche supérieure du lit de galets-eau repérée par l'indice 2), à la température du liquide, sont petites devant la pression totale $P$ du gaz, l'équation de Stefan peut se simplifier :

$$
m=\left(D_{\mathrm{c}} / l_{0}\right)\left(P_{2}-P_{\mathrm{v}}\right) S /\left(R_{\mathrm{v}} T\right) \quad \mathrm{kg}
$$

avec :

$$
R_{\mathrm{v}}=8,31 \quad \mathrm{~J} \text { mole }{ }^{-1} \mathrm{~K}^{-1}
$$

$R_{\mathrm{v}}$ : constante des gaz parfaits

$h_{\mathrm{m}}=D_{\mathrm{c}} / l_{0}$ est un coefficient de transfert de masse. Il est obtenu par analogie entre les transferts calorifiques et massiques.

Plusieurs études concernant des configurations expérimentales semblables à celle du dispositif de la toiture diode [16-20] ont montré que la fonction de Lewis pouvait être prise égale à 1 :

$$
f(L e)=h_{\mathrm{c}} /\left(h_{\mathrm{m}} \rho C_{\mathrm{p}}\right)
$$

avec :

$\rho$ : masse volumique du $\mathrm{gaz} \mathrm{kg} \mathrm{m}^{-3}$

$C_{\mathrm{p}}$ : chaleur spécifique du gaz $\mathrm{J} \mathrm{kg}^{-1} \mathrm{~K}^{-1}$

$h_{\mathrm{c}}$ : coefficient d'échange convectif $\mathrm{W} \cdot \mathrm{m}^{-2} \mathrm{~K}^{-1}$

On retrouve ainsi la relation de Clapeyron (21) pour le flux évaporé $\phi_{\mathrm{e}}$ :

$$
\phi_{\mathrm{e}}=1,67 h_{\mathrm{c}}\left(P_{2}-P_{\mathrm{v}}\right) S \quad \text { W.m } \mathrm{m}^{-2}
$$

$P_{2}$ et $P_{\mathrm{v}}$ étant en millibars.

La condensation n'est pas exactement le phénomène inverse de l'évaporation en raison de la situation géométrique opposée du condenseur relativement à celle de l'évaporateur, et de la présence d'un condensat à éliminer au fur et à mesure de sa formation.

Lorsque les deux processus d'évaporation et de condensation sont dépendants l'un de l'autre, d'autres paramètres remplacent ceux rencontrés dans le cas de l'évaporation d'un film d'eau horizontal : l'épaisseur de la couche limite se trouve remplacée par la distance $d$ entre les échangeurs; la température du gaz disparaît au profit de la température du condenseur. L'expression de $\phi_{\mathrm{e}}$ reste semblable, $P_{\mathrm{v}}$ étant remplacée par la pression de vapeur saturante $P_{3}$ au condenseur, et $h_{\mathrm{c}}$ devenant le coefficient d'échange global ; $h_{\mathrm{c}}$ est obtenu à partir du nombre de Nusselt :

$$
N_{\mathrm{u}}=h_{\mathrm{c}} d / \lambda_{\mathrm{a}}
$$

où $\lambda_{\mathrm{a}}$ est la conductivité du mélange gaz-vapeur W. $\mathrm{m}^{-1} \mathrm{~K}^{-1}$.

3.2 RÉSultats EXPÉRIMENTAUX. - Pour étudier en régime stationnaire les transferts thermiques au travers du système de toiture diode, le dispositif représenté sur la figure 8 a été réalisé :

- Une enceinte d'expérimentation contenant un peu d'eau est constituée par un tronc de cylindre en 


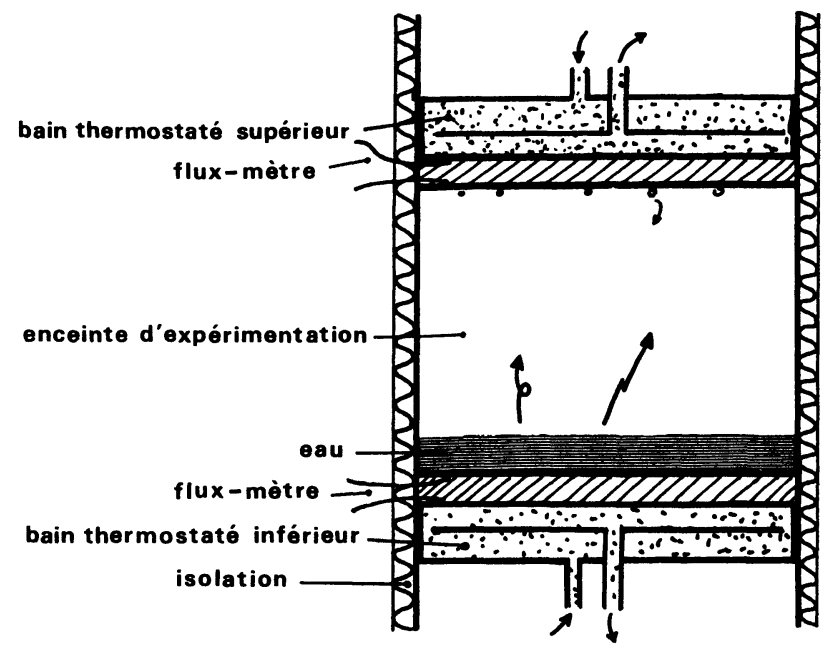

Fig. 8. - Schéma de principe du dispositif de laboratoire réalisé pour l'étude du processus caloduc : enceinte cylindrique fermée par deux pistons thermostatés.

[Skeleton diagram of the system built to study the heatpump process : cylindrical volume closed with two heated pistons.]

P.V.C. de $0,5 \mathrm{~m}$ de diamètre, isolé sur le pourtour. Supérieurement et inférieurement on trouve : un flux-mètre fait de deux plaques de dural séparées par un disque de nylon de $1 \mathrm{~cm}$ d'épaisseur. Sur chaque plaque sont serties 3 sondes thermostatiques.

- Un bain thermostaté alimenté par une cuve extérieure thermostatée. La distance entre fluxmètres a été variable et l'absorbeur supérieur a eu intérieurement des formes diverses pouvant plus ou moins faciliter l'écoulement du condensat.

Les principaux résultats observés ont été les suivants : le nombre de Nusselt obtenu par Ralph et al. [22] semble bien correspondre à la disposition du système, alors que la corrélation donnée par Graaf ne paraît pas convenir (Fig. 9). La température du mélange air-vapeur est inférieure de $1^{\circ} \mathrm{C}$ à la moyenne arithmétique des températures de l'évaporateur et de l'absorbeur :

$$
T_{\text {air confiné }}=1 / 2\left(T_{2}+T_{3}\right)-1 .
$$

La condensation se présente donc comme un processus plus efficace que l'évaporation dans la configuration du système. La figure 10 montre l'aspect « diode » et l'importance des flux qui peuvent être obtenus. Les flux parasites latéraux ont été pris en compte grâce à la présence des deux flux-mètres. Comme le prévoit la théorie, le système est d'autant plus performant que les températures sont élevées (climats chauds).

D'autres résultats concernent la distance $d$, la masse thermique, l'importance du transfert radiatif [23].

Finalement, on observe que pour des écarts de température $\Delta T$ entre échangeurs inférieurs à $10^{\circ} \mathrm{C}$,

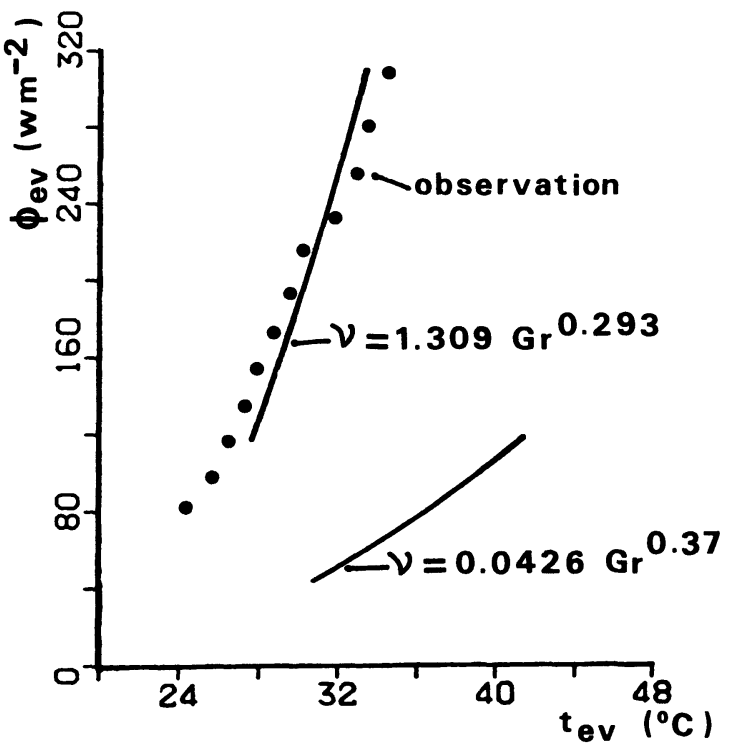

Fig. 9. - Flux évaporé, observé, et théorique pour deux corrélations de Nusselt, en fonction de la température à l'évaporateur.

[Evaporated fluxes, observed and theoretical for two Nusselt correlations, as functions of the evaporator temperature.]

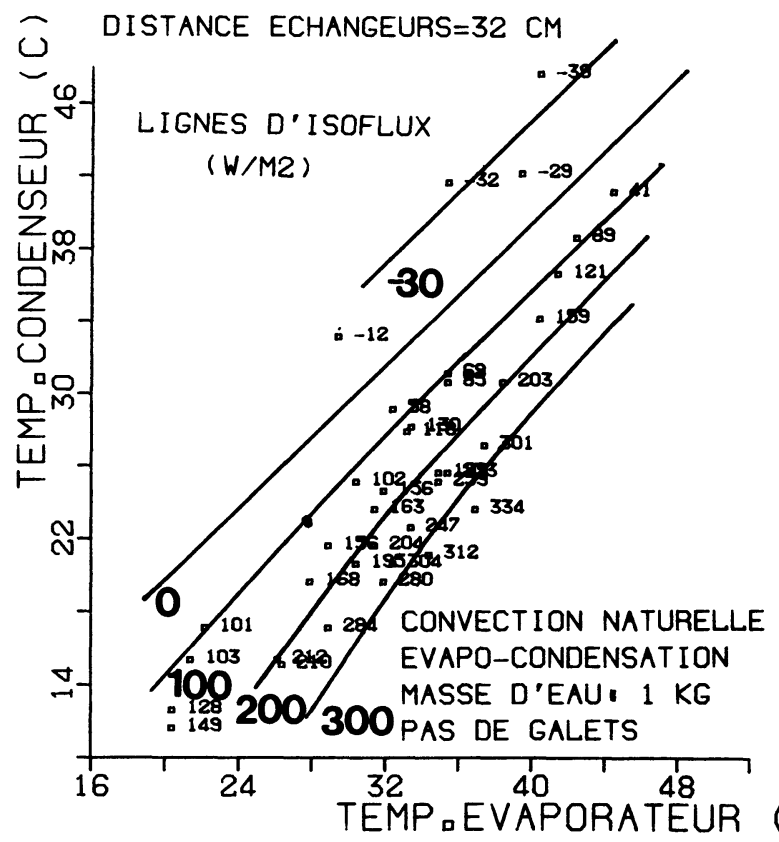

Fig. 10. - Energie transférée observée en fonction des températures à l'évaporateur et au condenseur : mise en évidence de l'effet « diode».

[Observed transferred energy as a function of evaporator and condenser temperatures : view of the « diode » effect.]

la théorie considérée sous-estime l'expérience de 5 à $20 \%$, ce qui peut se corriger en adoptant pour Nusselt :

$$
N_{\mathrm{u}}=1,33 \text { Grashof }^{0,3008} \text {. }
$$


Pour $T=10^{\circ} \mathrm{C}$, la sur-estimation conduit à adopter pour Nusselt :

$$
N_{\mathrm{u}}=1,30 \text { Grashof }^{0,2933} .
$$

\section{Comportement thermique de la toiture diode.}

Sur la base des équations ci-dessus établies, des simulations sur 48 heures ont été effectuées sur ordinateur pour étudier le comportement thermique du dispositif dans un contexte climatique donné, ainsi que l'influence des paramètres. La réalisation d'une cellule-test, véritable petite maison de $15,6 \mathrm{~m}^{3}$, a permis par ailleurs d'observer la réalité de ce comportement.

4.1 RÉSUltats THÉORIQUES. - La puissance radiative céleste étant faible, il importe que l'habitat soit fortement isolé ( $\dot{Q}_{\mathrm{d}}$ et $\dot{Q}_{1}$ petits dans l'équation (1) pour limiter les apports calorifiques) et que les apports solaires absorbés soient fortement limités ( $\alpha$ petit), étant donnés d'une part l'ordre de grandeur supérieur de la puissance radiative solaire, d'autre part le fait que la toiture ne représente que la seule des 6 faces de l'habitat pour l'action de refroidissement. L'adaptation d'un support à recevoir un

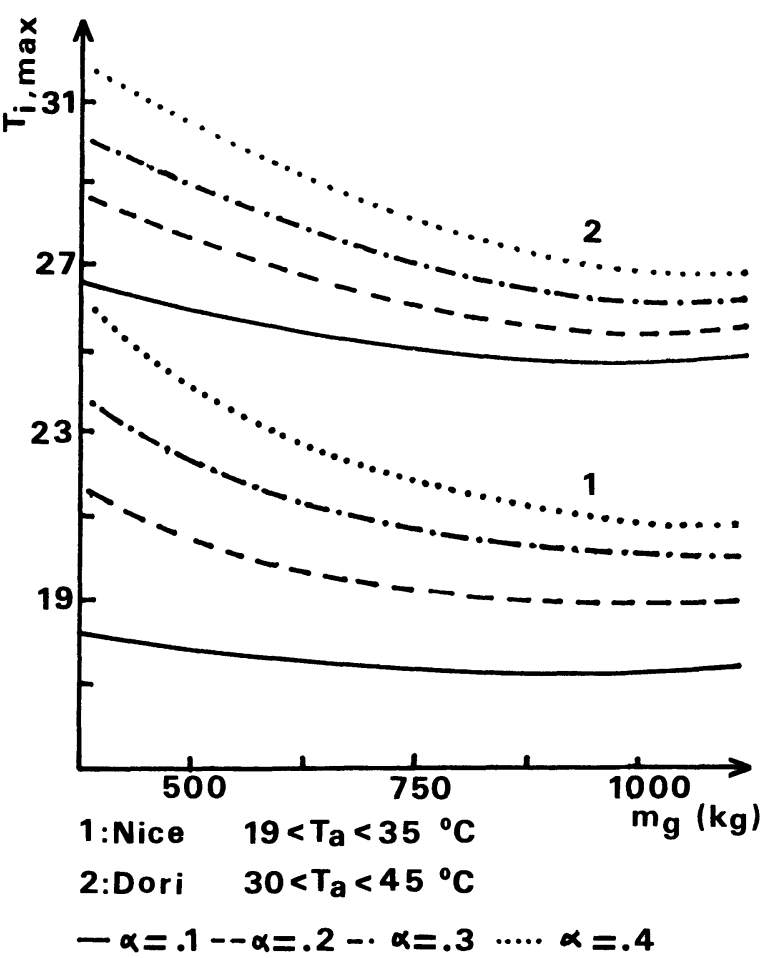

Fig. 11. - Influences du coefficient d'absorption $\alpha \mathrm{du}$ rayonnement solaire et de la masse inertielle (pour $6,25 \mathrm{~m}^{2}$ de toiture) sur la température intérieure maximale ; pour $\alpha$ nul elle est indifférente à la masse.

[Influences of the $\alpha$ coefficient of solar radiation absorption, and of the inertial mass (for a $6.25 \mathrm{~m}^{2}$ roof) on the maximum inside temperature. When $\alpha$ is null, this temperature is indifferent to the mass.] dispositif est toujours la première action à effectuer. L'isolation, convective et radiative, représente dans le cas présent, l'adaptation à obtenir. La figure 11 montre l'intérêt et la nécessité d'une forte masse thermique pour limiter l'élévation de la température maximale intérieure de l'habitat, et d'un coefficient $\alpha$ faible. Lorsque les apports solaires sont de même importance que les pertes radiatives vers la voûte céleste $(\alpha \not 0,1)$, la température maximale est alors indépendante de la masse inertielle. En général, l'absorptivité des peintures blanches disponibles dans le commerce est supérieure à 0,2 ; elle justifie l'importante masse de galets et d'eau nécessaire à la limitation du réchauffement solaire de la toiture de l'habitat. Le déphasage entre les moments où les températures sont maximales à l'intérieur et à l'extérieur augmente lorsque $\alpha$ diminue (Fig. 12). L'inté-

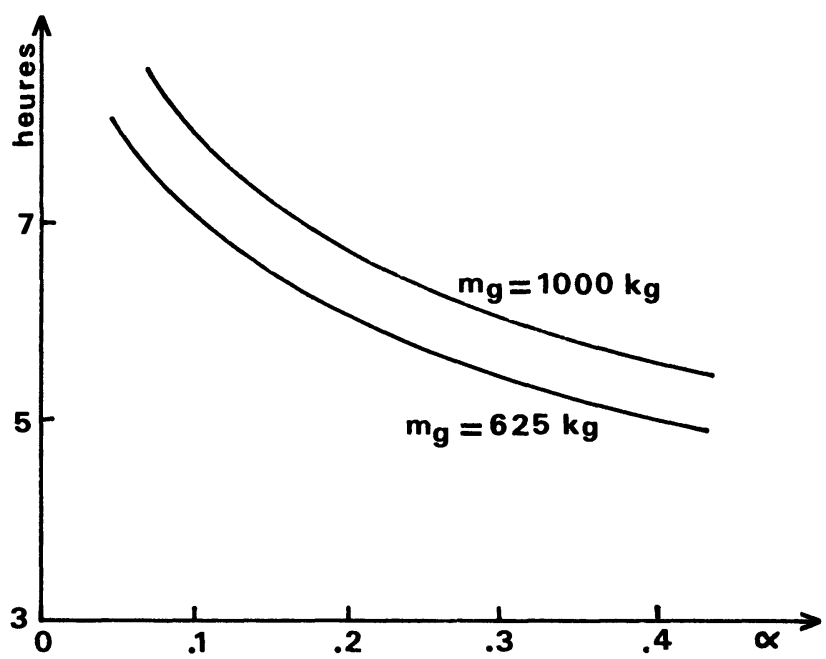

Fig. 12. - Déphasage entre les moments où les températures d'air intérieure et au dehors sont maximales, en fonction du coefficient $\alpha$ d'absorption du rayonnement solaire et de la masse inertielle (pour $6,25 \mathrm{~m}^{2}$ de toiture).

[Phase shift between the times when inside and outside air temperatures are maximum, as a function of $\alpha$ and of the inertial mass (for a $6.25 \mathrm{~m}^{2}$ roof).]

rêt d'un déphasage d'1/3 jour et non de 2 à 3 heures réside dans la possibilité offerte en début de nuit de faire appel à la ventilation pour un refroidissement complémentaire convectif (ce refroidissement a son origine radiative par l'intermédiaire de l'environnement ; d'où l'importance d'un environnement thermiquement favorable : fond de vallée...). L'action de refroidissement n'ayant pas lieu dans la journée, il importe d'une part de limiter les apports calorifiques, d'autre part d'avoir pu s̄tocker des frigories.

L'intégration numérique effectuée pour le cas d'un climat très chaud et sec (Fig. 13) montre que la température intérieure reste au voisinage de la température minimale nocturne (amplitude : $3^{\circ} \mathrm{C}$ ). 
En début de nuit, la température d'air intérieur étant légèrement supérieure à celle de l'air extérieur, on retrouve l'intérêt d'une ventilation de l'habitat comme processus complémentaire pour maintenir la température intérieure minimale. Dans la journée, le refroidissement évaporatif (jarre suintante, linge humide...) est l'action complémentaire souhaitable. Les températures obtenues dans l'habitat (Fig. 13) sont à peine supérieures aux températures souhaitées [10].

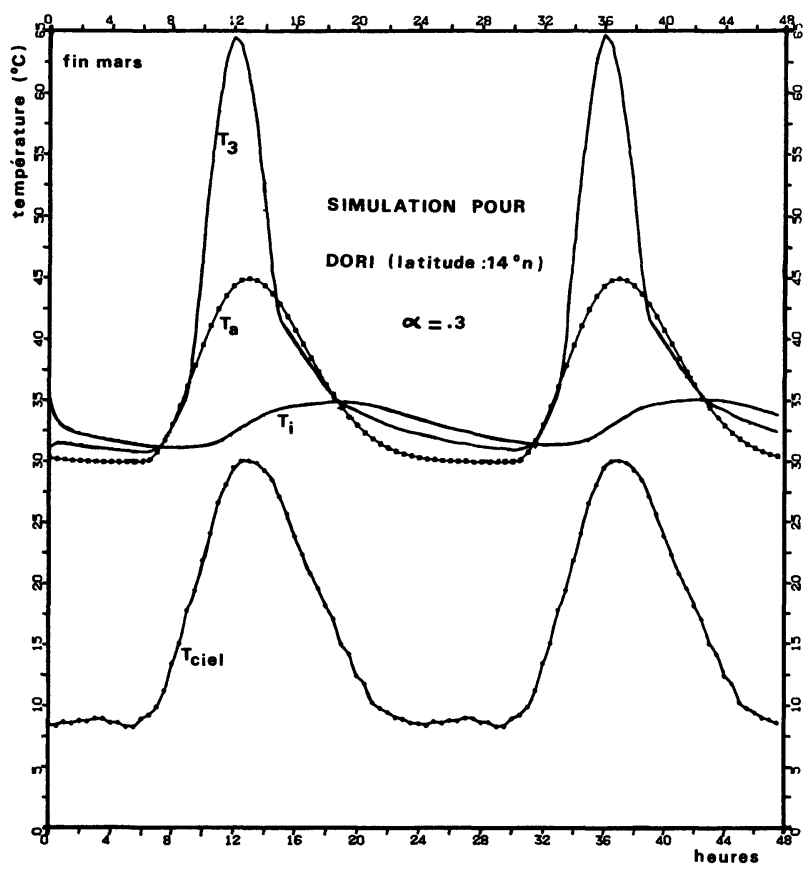

Fig. 13. - Simulation de fonctionnement de la cellule-test pendant un cycle de 48 heures : $T_{\mathrm{i}}$ varie avec une amplitude faible quelques degrés au-dessus de $T_{\mathrm{a}, \min }$.

[Simulation of the cell test running during a two day cycle : $T_{\mathrm{i}}$ fluctuates with a small amplitude a few degrees over $\left.T_{\mathrm{a}, \min }.\right]$

D'autres simulations sur ordinateur ont été faites elles ont par exemple montré l'intérêt des stores fixes ou mobiles pour protéger l'absorbeur supérieur lorsque $\alpha$ n'est pas petit [14].

\subsection{EXPÉRIMENTATION AVEC UNE CELLULE-TEST.}

- Avant de réaliser dans un pays à climat chaud et sec une maison à toiture-diode, il était indispensable d'en expérimenter une dans un site propice aux études scientifiques. La région niçoise n'est pas idéale dans la mesure où son climat est de type méditerranéen : humidité élevée, nuits courtes, amplitudes thermiques faibles, températures moyennes peu élevées. C'est pourquoi les résultats observés avec la cellule-test construite près de Nice ne peuvent être que moins bons relativement à ceux qui seraient obtenus en pays sahélien. La figure 14 présente une

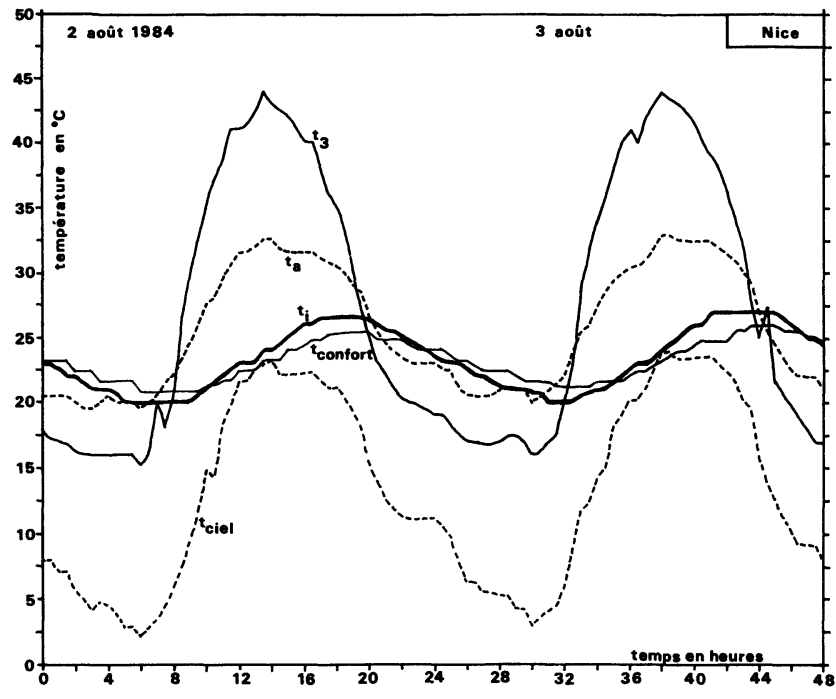

Fig. 14. - Températures enregistrées pendant 48 heures pour la cellule-test: le rayonnement du plafond est bénéfique à la température de confort.

[Observed temperatures during 48 hours in the cell test : the ceiling radiance is a profit to the comfort temperature.]

partie des températures observées les 2 et 3 août 1984. La température équivalente de rayonnement du ciel a été mesurée à l'aide d'un pyrgéomètre Eppley, et la température de confort à l'intérieur de la cellule-test à l'aide d'un P.M.V.-mètre Brüel et Kjaer. Pour ces journées, l'hygrométrie moyenne était de $60 \%$. La sensation de confort ressentie correspond à celle qui aurait été souhaitée par plus de $95 \%$ d'occupants.

L'écart entre la température intérieure d'air et celle de confort témoigne de l'action radiative bénéfique des parois. La valeur moyenne et l'amplitude de ces températures auraient été plus basses si la durée de la nuit avait été plus longue, ce qui a été observé en septembre. Pour le reste, les résultats obtenus par les simulations sur ordinateur ont été confirmés : déphasage temporel...

\section{Conclusion.}

Le présent article n'est qu'une présentation très abrégée des travaux effectués pendant plus de 3 ans, et qui ont abouti à deux thèses et plusieurs publications dans des congrès. La présentation faite ici a pour but de faire connaître ces travaux, mais requiert un report du lecteur à ces ouvrages.

Le travail s'est appuyé sur des études nouvelles concernant le confort thermique de l'être humain et le rayonnement atmosphérique. Pour des régions où le climat est une contrainte permanente, la solution thermique passe par une utilisation judicieuse de plusieurs modes d'échanges thermiques, soit en une association ou en une opposition, soit en une complémentarité temporelle. 
L'intérêt du dispositif de toiture diode, qui reste à confirmer par une réalisation en climat chaud et sec, résulte de la prise en compte des exigences financières, des caractéristiques climatiques, et des performances possibles, mais limitées, de plusieurs systèmes.

L'habitat traditionnel est le résultat d'un équilibre atteint dans lequel le mode de vie s'est adapté à des contraintes thermiques ou autres, et à des possibilités locales climatiques et matérielles. La toiture diode, se présentant comme une toiture en tôle classique, peut être considérée comme un système traditionnel parce qu'elle ne rompt pas cet équilibre. Les connaissances scientifiques actuelles peuvent ainsi permettre de rendre plus performants les systèmes traditionnels. Les besoins en climatisation sont la plupart du temps momentanés, et les possibilités offertes faibles. C'est pourquoi, bien plus qu'en chauffage, le recours aux systèmes traditionnels se justifie: la réhabilitation et l'amélioration des techniques ancestrales peuvent être possibles et suffisantes, grâce aux connaissances scientifiques et techniques actuelles. C'est un objectif à poursuivre, et qui a été visé dans ce travail.

\section{Bibliographie}

[1] Awanou, C. N., Berger, X., A simple system for sky temperature measurements, Colloque Météorologie et énergies renouvelables, Valbonne (1984) p. 47-57.

[2] Treat, C. H., Allen, C., An assessment of rockbeds for comfort cooling in several U.S. cities, Third National Passive Solar Conference, San José California (1979) p. 452-456.

[3] Givoni, B., Passive cooling of buildings by natural energies, Energy Buildings 2 (1980) 1-7.

[4] YADAV, R., RAD, D. P., Digital simulation of indoors temperatures of buildings with roof ponds. Solar Energy 31 n $^{\circ} 2$ (1983) 205-215.

[5] JACOB, D., LOTZ, H., Refroidissement par rayonnement zénithal à Béni-Abbès. Revue Int. Héliotechn. COMPLES $2^{\text {ème }}$ trim. (1979) 23-29.

[6] Berger, X., Buriot, D., Garnier, F., About the equivalent temperature for clear skies. Solar Energy 32 (1984) 725-753.

[7] Al Jamal, K., QuinN, M., Jarrar, D., Shaban, N., YAGOUB, Y., SouZA, J. D., Dust effect on infrared sky radiation in Kuwait. Solar Wind Technol. 2 (1984) 109-114.

[8] Berger, X., Grivel, F., Deval, J. C., Le confort thermique en climat chaud. Rapport REXCOOP Ministère Plan-Construction $n^{\circ} 83.166$ Paris 1984.

[9] Berger, X., Grivel, F., Deval, J. C., Le confort thermique vécu en climat chaud. Rapport REXCOOP A.F.M.E. $n^{\circ}$ 43302326, Valbonne 1985.

[10] Humphreys, M. A., The dependence of comfortable temperatures upon indoor and outdoor climates, chapter in Bioengineering, thermal physiology and comfort edited by K. Céna and J. A. Clark (Elsevier SC pub. Co Amsterdam) 1981.
[11] McIntyre, D. A., Indoor Climate (Applied Science Publishers, London) 1980.

[12] Fanger, P. O., Ipsen, B. M., Langkiloe, G., OleSen, B. W., Christensen, N. R., Tanabé, S., Comfort limits for asymmetric thermal radiation. Energy Buildings 8 (1985) 225-236.

[13] C.S.T.B., Règles de calcul du coefficient $G$ des bâtiments Th K 77 DTU 1975.

[14] Awanou, C. N., Etude de réfrigération passive par toiture diode. Thèse doctorat $3^{\text {ème }}$ cycle Nice 1984, prix de thèse C.N.R.S. 1985.

[15] SACCADURA, J. F., Initiation aux transferts thermiques (Ed. Technique et Documentation) 1978.

[16] Tusseau, P., Gaz humides. Techn. Ingénieur 3 (1961).

[17] Landau, L., LifChitz, E., Mécanique des fluides (Ed. Mir Moscou) 1971.

[18] VACHON, M., Evaporation en convection naturelle. Revue Phys. Appl. 15 (1980) 427-431.

[19] SнAн, M. M., Estimation of evaporation from horizontal surface. ASHRAE fundamentals 1981.

[20] Bernard, R., Menguy, G., Schwartz, M., Le rayonnement solaire, conversion thermique et applications (Technique et Documentation, Paris) 1980.

[21] Pelletret, R., Berger, X. : Capteur climatique. Revue Phys. Appl. 18 (1983) 177-189.

[22] RALPH, J. C., Heat and mass transfer across gas filled enclosed spaces between a hot liquid surface and cold roof (Hemisphere Pub. Corp.) 1976, 497 508.

[23] Kieno, P. F., Etude théorique et expérimentale d'un dispositif caloduc pour climatisation en pays chauds et secs. Thèse doctorat $3^{\text {ème }}$ cycle, Nice 1985. 